\title{
Performance Estimation of Surface Acoustic Wave Motor Using Simulation Model of Friction Drive
}

\author{
Katsuhiko Asai ${ }^{1}$ and Minoru Kuribayashi Kurosawa ${ }^{2}$ \\ ${ }^{1}$ Advanced Technology Research Laboratories, Matsushita Electric Industrial Co., Ltd., Kyoto, 619-0237 Japan \\ ${ }^{2}$ Department of Advanced Applied Electronics, Tokyo Institute of Technology, Yokohama, 226-8502 Japan
}

SUMMARY

The contact states of the slider and stator substrate greatly affect the drive performance of a surface acoustic wave motor. The contact states are determined by factors such as the friction coefficient, vibration amplitude, rigidity of the contact surface, contact time with the Rayleigh wave, and slider speed. A model was proposed that represented the relationship between these factors and the drive performance. However, guidance has not been provided on how these values should be changed to improve the drive performance. In this research, we use a friction drive model we proposed to analyze the effect of the changes in these parameters on the drive performance and to discuss the motor design and the drive conditions based on evaluations from the perspectives of the drive power, output, and efficiency. () 2004 Wiley Periodicals, Inc. Electron Comm Jpn Pt 3, 88(1): 37-47, 2005; Published online in Wiley InterScience (www.interscience.wiley.com). DOI 10.1002/ ecjc.20079

Key words: surface acoustic wave; actuator; friction drive; contact analysis; MEMS.

Contract grant sponsor: Grant-in-Aid for Scientific Research from the Ministry of Education, Culture, Sports, Science and Technology.

\section{Introduction}

A surface acoustic wave motor is an actuator that produces drive power through frictional force by a Rayleigh wave, which is a type of surface acoustic wave. Therefore, to improve the performance of a surface acoustic wave motor, the deformation of the contact surface and the stickslip state must be understood, and the motor design and drive conditions must be selected for a drive state suited to the objective. However, no guidance has been reported concerning these issues. The projection arrangement on the slider and drive conditions were selected based on experimental results.

Since the state of the contact surface is difficult to understand analytically, the authors previously proposed a friction drive model that considers the changes in the stick and the slip states and demonstrated its effectiveness by comparing the test results for a ball slider and a silicon slider $[1,2]$. By using the proposed friction drive model, we showed the changes of the stick and slip states over time based on the friction coefficient, vibration amplitude, rigidity of the contact surface, contact time with the Rayleigh wave, and slider speed, and determined the force added to the projections of the slider at each time. From this friction drive model, we can estimate the effect of changes in the drive conditions on the drive performance and the limiting performance. Various reports attempted to understand the contact states of traveling ultrasonic wave motors, which have a similar drive principle, by using methods such as theoretical analysis, finite element methods, or numerical analysis based on simplified friction drive models [3-13].

(C) 2004 Wiley Periodicals, Inc. 
However, there have been almost no reports on the design guidelines or the drive condition settings corresponding to the objective.

In this paper, we apply the friction drive model proposed by the authors to analyze the effect of changes in each parameter on the drive performance and discuss the motor design and drive conditions based on evaluations from the perspectives of the drive force, output, and efficiency.

\section{Overview of the Friction Drive Model}

This section presents an overview of the friction drive model we proposed in Ref. 1 . This model basically determines the changes in the force received by one projection and the slip distance. The eight values needed for the calculations based on the friction drive model are the drive angular frequency $\omega$; friction coefficient $\mu$; vibration amplitudes $a_{v}, a_{h}$; rigidity $k_{v}, k_{h}$ of the contact surface; normal slider position $y_{s}=a_{v}$ sin $\phi$; and slider speed $v=a_{h} \omega \sin$ $\psi$. The subscripts $v$ and $h$ of the amplitudes and rigidity indicate the normal and tangential directions with respect to the substrate surface.

The vibration displacement $y$ in the normal direction and the vibration displacement $x$ in the tangential direction of the Rayleigh wave are represented as follows for one period $(-\pi / 2 \leq \omega t \leq 3 \pi / 2)$ :

$$
\begin{aligned}
& y=a_{v} \sin \omega t \\
& x=-a_{h} \cos \omega t
\end{aligned}
$$

For constant $\phi$ and $\psi$, the time when a projection is in contact with the Rayleigh waves is represented by $\phi \leq \omega t \leq$ $\pi-\phi$. The elastic force $f_{n}$ in the normal direction received by this projection becomes

$$
f_{n}=k_{v} a_{v}(\sin \omega t-\sin \phi)
$$

The driving force $f$ in the tangential direction added to the projection is expressed by

$f=k_{h}\left\{a_{h}(\cos \phi-\cos \omega t-(\omega t-\phi) \sin \psi)+d_{s}\right\}$

where $k_{h}$ multiplies the sum of the relative distance from the point where the projection is initially in contact to the projection position and the displacement due to slip. $d_{s}$ is the function that represents the displacement due to slip. This function has the initial value of 0 and changes when $|f|$ $>\mu f_{n}$ and slip occurs, and takes the value satisfying $|f|=$ $\mu f_{n}$ at this time. On the other hand, the value does not change in the stick state of $|f| \leq \mu f_{n}$. Here, $\mu$ denotes the friction coefficient, and for simplicity, the static friction coefficient and the dynamic friction coefficient are equal. The power extracted by the friction drive is $p=f \cdot v$. The slip loss $l$ at the friction surface is determined by the product of the friction force and the slip speed and is found to be

$$
l=\left|\mu f_{n} \cdot \frac{d}{d t}\left(d_{s}\right)\right|
$$

The calculation described above can analyze the contact of the surface acoustic wave motor by making a decision about stick or slip while advancing at each small time step. Although the static friction coefficient and the dynamic friction coefficient are equal, by distinguishing between the decisions of "stick $\rightarrow$ slip" and "slip $\rightarrow$ stick," the analysis can also be easily performed when the static friction coefficient and the dynamic friction coefficient differ. Figure 1 is an example of the time variation calculations of $f, d_{s}$, and l. The result is normalized, and $\alpha=\mu k_{v} a_{v} /\left(k_{h} a_{h}\right), F_{a}=$ $\mu\left(k_{v} a_{v}(2 \pi)\right.$, and $v_{0}=a_{h} \omega$. In this example, after the projection initially comes into contact with the stator substrate, the states change as slip $\rightarrow$ stick $\rightarrow$ slip $\rightarrow$ stick $\rightarrow$ slip. In the slip state, $d_{s}$ changes, and slip loss is produced. In this friction drive model, as is clear from Eqs. (3) and (4), the stick and slip generation states in one period depend on three parameters, $\alpha, \phi$, and $\psi$. $\alpha$ denotes the stick tendency. The stator substrate and projection always stick when $\alpha=$ $\infty$ and always slip when $\alpha=0$.

In the friction drive model, when the changes in the force during one period are determined and the performance as a motor is considered, the time average per period may be used as the value in the steady state. The time averages per period of $f_{n}, f, p$, and $l$ are defined as the preload
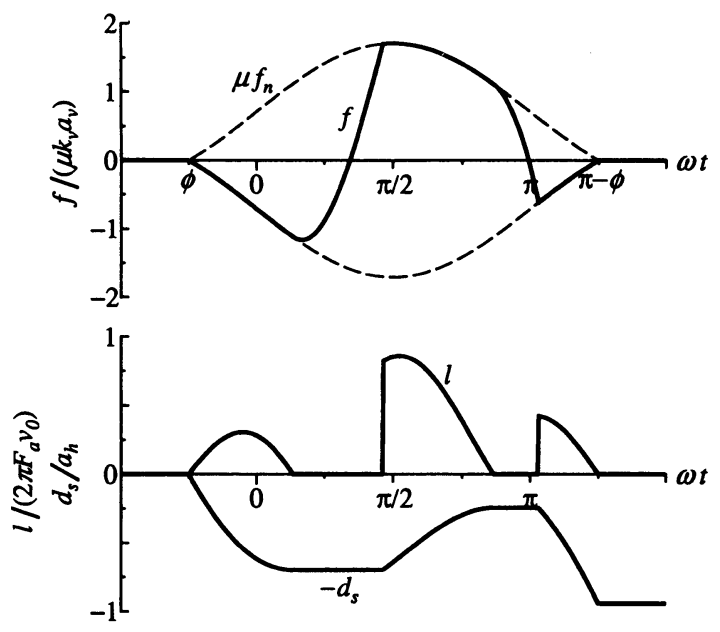

Fig. 1. Example of time variations of $f, d_{s}, l(\alpha=0.1, \phi$

$$
=-\pi / 4, \psi=\pi / 6) \text {. }
$$


$N$, driving force $F$, output power $P$, and slip loss $L$. When the slider has multiple projections, the changes in the contact surface rigidity due to the projection arrangement are considered, and the force received by each projection is integrated.

\section{The Effect of Changes in the Drive Conditions on the Drive Performance}

When the friction drive model is used, eight values are required to calculate the drive performance of a surface acoustic wave motor. Five of these parameters $\omega, a_{v}, a_{h}, \phi$, and $\psi$ are values determined by the drive conditions of the motor. The remaining parameters $\mu, k_{v}$, and $k_{h}$ are values determined by the materials of the stator substrate and the slider, and the shape and arrangement of the slider projections, and become the design parameters when designing the surface acoustic wave motor. When the motor design and drive conditions are selected, how the drive performance varies based on these values must be clarified. However, determining the individual effect of all eight becomes difficult. The drive performance is represented by the three values of $\alpha=\mu k_{v} a_{v} / k_{h} a_{h}, \phi$, and $\psi$ by expressing the force, speed, and time by normalized values. Instead of determining the effect of each value on the drive performance, the changes in the drive performance due to $\alpha$ are determined. In the slider used in previous research, a ball slider is considered to be suited to $\alpha=0.2$ [1]. For a silicon slider, $\alpha$ is thought to be from 1 to 5 based on estimates from experimental results [2].

\subsection{The relationship between driving force and slider speed}

Figures 2 to 4 show the initial calculation of the relationship between the driving force $F$ and the slider speed $v$. The figures show the results for $\alpha=0.1,1$, and 10, respectively. The seven lines in the figures indicate the differences when the value of $\phi$ changes. The driving force and the slider speed are normalized by $F_{a}=\mu k_{v} a_{v} / 2 \pi$ and $v_{0}=a_{h} \omega . \phi$ takes a value from $-\pi / 2$ to $\pi / 2$. In the state where $\phi=\pi / 2$, the projections are in contact only with the wave crest of the Rayleigh wave. In the state where $\phi=-\pi / 2$, the projections are in contact with the wave floor of the Rayleigh wave and always in contact with the stator substrate. Thus, the preload decreases as $\phi$ increases, and the contact time becomes short.

From Figures 2 to 4, the normalized 0 speed driving force increases as $\alpha$ decreases. This trend is conspicuous as $\phi$ becomes small. As $\alpha$ decreases, the decrease in the no-load speed becomes small as $\phi$ decreases. These show that for the same friction coefficient, amplitude of the Rayleigh wave, and rigidity in the normal direction, the 0

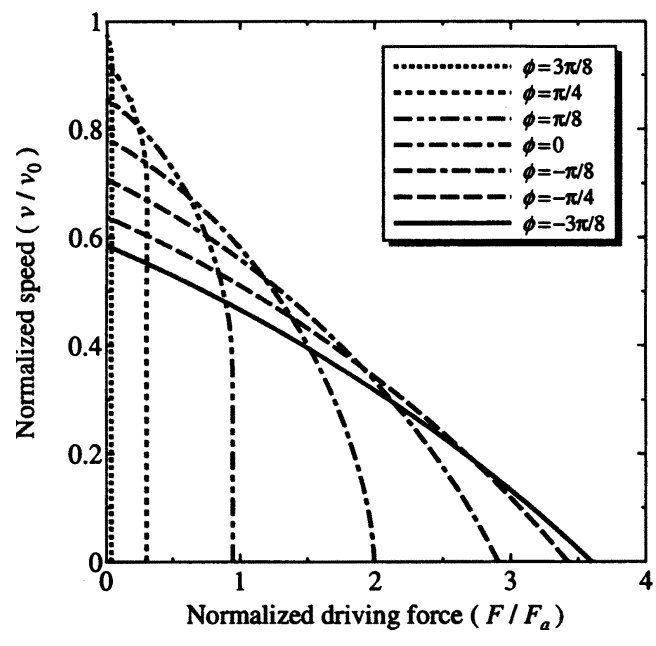

Fig. 2. Driving force-speed curves $(\alpha=0.1)$.

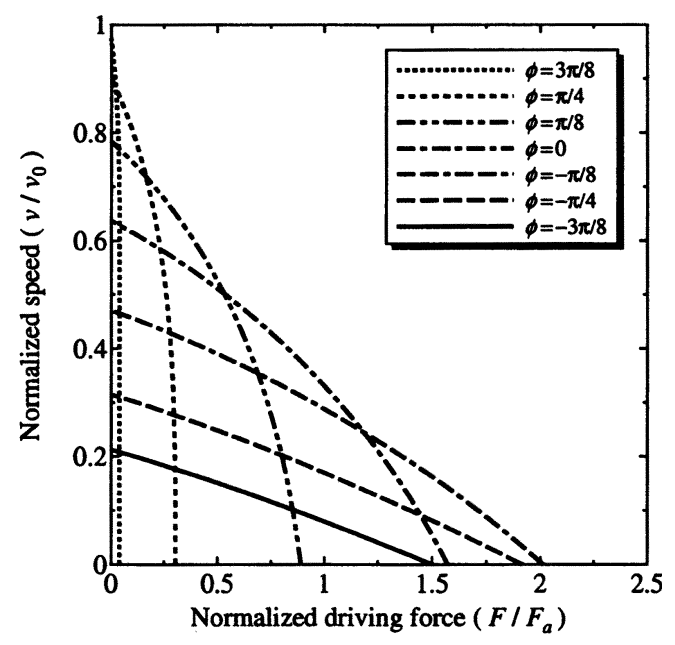

Fig. 3. Driving force-speed curves $(\alpha=1)$.

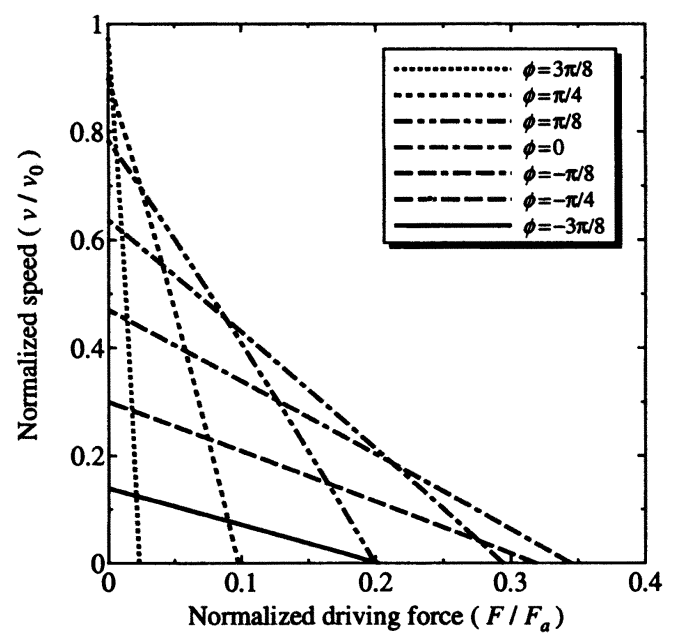

Fig. 4. Driving force-speed curves $(\alpha=10)$. 
speed driving force and the no-load speed increase as the rigidity in the tangential direction increases. The appearance of the drooping characteristic changes linearly when $\alpha=10$. As $\alpha$ decreases, the change in the driving force as the speed changes depends on the speed. In particular, when $\phi$ is large, a speed range exists where the driving force does not change. This is a state where slip is produced in the entire contact range. These characteristics are exhibited because the changes in the friction force due to the slip speed are not produced by Coulomb friction. Since the driving force changes in the transient response decrease under these conditions, we know that the 0 speed driving force $F_{v=0}$ becomes somewhat larger than the estimate by the friction drive model when this force is determined by fitting to the equation of the transient response

$$
v=v_{F=0}\left(1-e^{-\frac{t}{\tau}}\right), \quad \tau=m \frac{v_{F=0}}{F_{v=0}}
$$

under the assumption of the relationship for the drooping characteristic of $v=v_{F=0}\left(1-F / F_{v=0}\right)$. Here, $v_{F=0}$ is the no-load speed. The ball slider is more easily affected than the silicon slider by the value of $\alpha$.

\subsection{The relationship between driving force and output power}

Figures 5 to 7 show the calculations of the relationship between the driving force $F$ and the output power $P$. In these figures, the output power $P$ is normalized by $P_{a}=$ $F_{a} v_{0}$. The value of the normalized output power increases as $\alpha$ decreases similar to the normalized 0 speed driving force. The maximum output power for each $\phi$ increases to some point when $\phi$ decreases, that is, when the preload

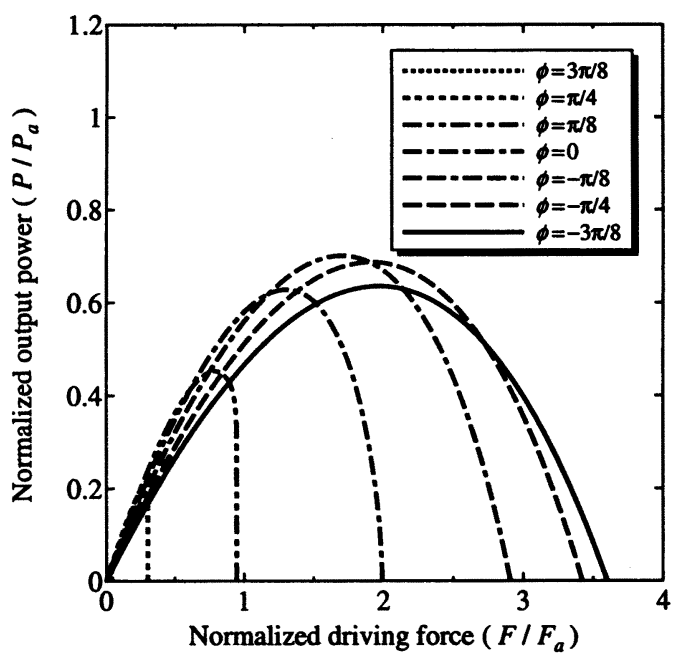

Fig. 5. Driving force-output power curves $(\alpha=0.1)$.

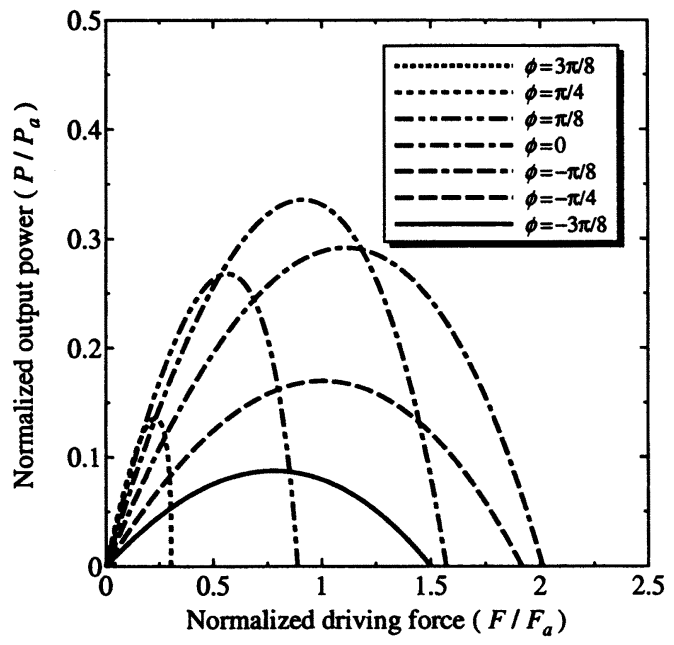

Fig. 6. Driving force-output power curves $(\alpha=1)$.

increases, and then reverses and decreases. We see from this that an optimum preload also exists in the output power as for the 0 speed driving force. The output power reaches its maximum when the driving force is nearly half its maximum value for $\alpha=10$ where the driving force-speed curve varies linearly, and reaches its maximum when the driving force is closer to the maximum driving force for $\alpha=0.1$ and 1 where the driving force-speed curve is no longer linear. This trend is conspicuous for a small $\alpha$.

We now determine the maximum driving force and maximum output power for each $\alpha$. The maximum driving force is determined when the slider speed $v$ is set to 0 and $\phi$ is varied. The maximum output power is determined when

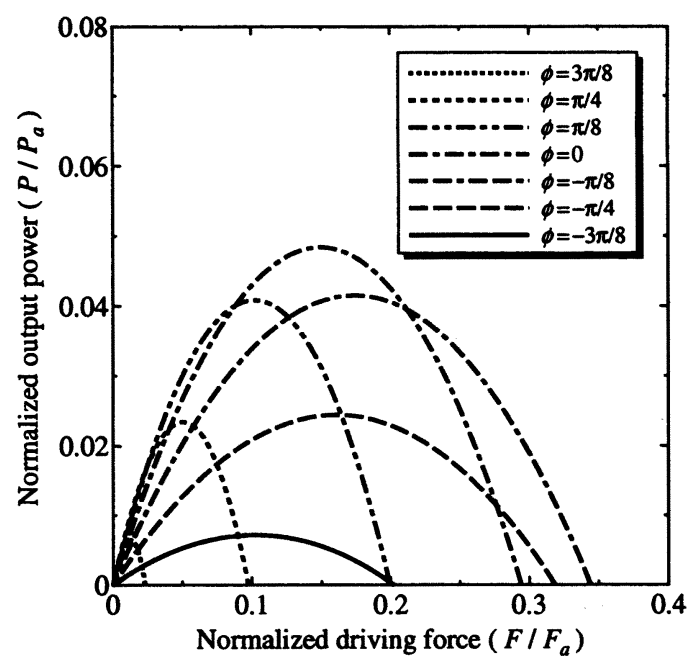

Fig. 7. Driving force-output power curves $(\alpha=10)$. 
both $\phi$ and the normalized slider speed $v / v_{0}$ are varied. Figure 8 shows the maximum driving force and the maximum output power which were determined. Figure 9 shows the $\phi$ in this case. We see from Fig. 8 that the normalized maximum driving force and the normalized maximum output power increase as $\alpha$ decreases. However, in the $\alpha>10$ region, both vary inversely proportional to $\alpha$. In the small $\alpha$ region, there are few changes and they converge to a constant as $\alpha \rightarrow 0$. The $\phi$ where each maximum occurs is nearly constant above the range $\phi=0.4$ to 0.6 . The maximum driving force occurs in the range $\phi=-\pi / 6$ to $-\pi / 7$, and the maximum output power occurs at $\phi=0$. Conversely, below the range $\phi=0.4$ and 0.6 , $\phi$ gradually decreases, and the Rayleigh wave must be further deformed to obtain the maximum values.

Based on the results in Fig. 8, we examine how the maximum driving force and the maximum output power vary when each parameter is varied. First, the rate of change of the normalized maximum driving force $F_{\max } / F_{a}$ with respect to $\alpha$ is defined as follows:

$$
\text { Rate of change for } \sqrt{\alpha_{n} \cdot \alpha_{n+1}}:\left(\frac{F_{n+1}^{\prime}}{F_{n}^{\prime}}\right)^{\frac{\frac{1}{\log _{10}\left(\frac{\alpha_{n+1}}{\alpha_{n}}\right.}}{(7)}}
$$

$\alpha_{n}$ and $F_{n}^{\prime}$ indicate the $\alpha$ and $F_{\max } / F_{a}$ for the $n$-th data point shown in Fig. 8, and $n$ becomes a low number as $\alpha$ decreases. In other words, $n=1,2, \ldots, 32$ correspond to $\alpha=$ $0.01,0.02, \ldots, 100$. The rate of change indicates the slope of the line connecting the $n$-th data point in Fig. 8 to the ( $n$ +1 )-st data point and becomes a power of $F_{\max } / F_{a}$ corresponding to 10 times $\alpha$ on this line. For example, the dashed lines in Fig. 8 have a slope equivalent to a rate of change of

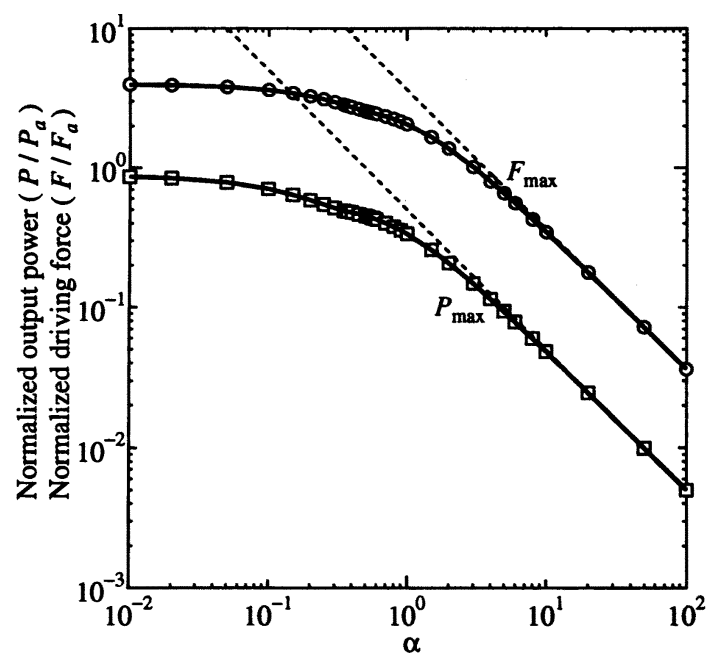

Fig. 8. Maximum driving force and maximum output power.

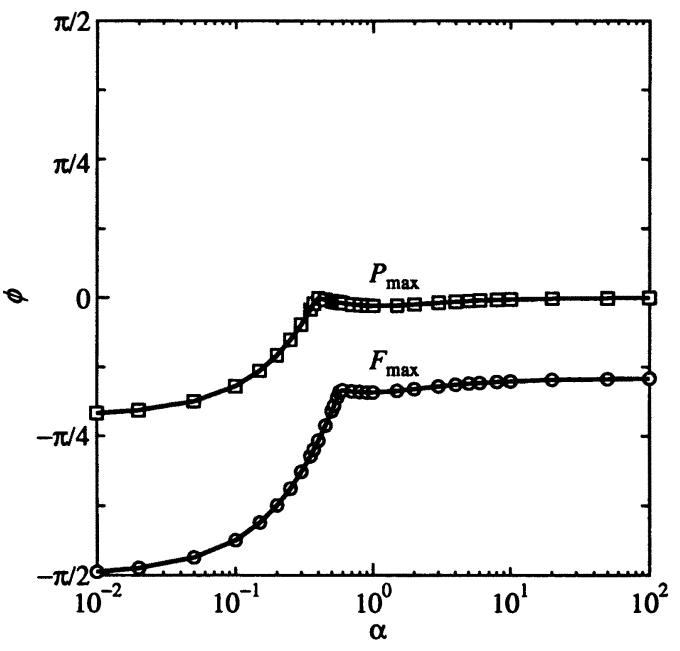

Fig. 9. $\phi$ at maximum driving force and maximum output power.

0.1 ( $-20 \mathrm{~dB} / \mathrm{dec}$.) because $F_{\max } / F_{a}$ is multiplied by 0.1 when $\alpha$ is multiplied by 10 . Figure 10 shows the calculation of the rate of change of $F_{\max } / F_{a}$.

The result in Fig. 10 always has a rate of change of 0 $\mathrm{dB} / \mathrm{dec}$. or less. A smaller $\alpha$ is preferred because the normalized maximum driving force $F_{\max } / F_{a}$ increases. However, if we consider an increase in the maximum driving force $F_{\max }$, we must consider $F_{\max } / F_{a}$ is the value normalized by $F_{a}=\mu k_{v} a_{v} /(2 \pi)$. For $\alpha=\mu k_{v} a_{v} /\left(k_{h} a_{h}\right)$, we consider multiplying each of $\mu, k_{v}$, and $a_{v}$ by 10 . In this case, since both $\alpha$ and $F_{a}$ are multiplied by 10, the rate of change of $F_{\text {max }}$ with respect to $\mu, k_{v}$, and $a_{v}$ becomes 10 times the rate

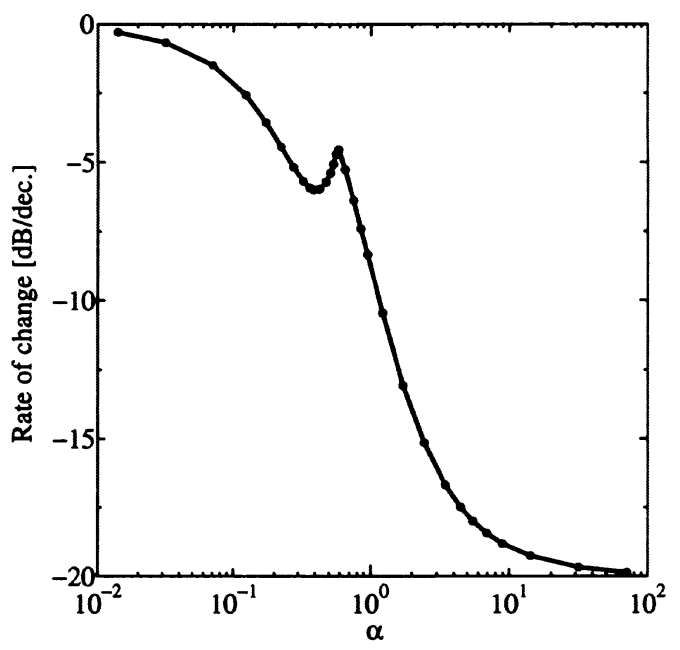

Fig. 10. Rate of change of $F_{\max } / F_{a}$. 
of change of $F_{\max } / F_{a}$ with respect to $\alpha$. On the other hand, for $k_{h}$ and $a_{h}$, since $F_{a}$ does not change and $\alpha$ is multiplied by $0.1, F_{a}$ becomes the reciprocal of the rate of change of $F_{\text {max }} / F_{a}$ with respect to $\alpha$. Figure 11 shows the rate of change of $F_{\max }$ determined above with respect to each value of $\mu, k_{v}, k_{h}, a_{v}$, and $a_{h}$ for each $\alpha$. From this result, we obtain the following knowledge about the maximum driving force.

- Since the rates of change for all $\alpha$ 's are at least 0 $\mathrm{dB} / \mathrm{dec}$., the maximum driving force always increases with increases in $\mu, k_{v}, k_{h}, a_{v}$, and $a_{h}$.

- Since the sum of the rates of change corresponding to $k_{v}$ and $k_{h}$ is always $20 \mathrm{~dB} / \mathrm{dec}$. for all $\alpha$, the rate of change of the maximum driving force is always $20 \mathrm{~dB} /$ dec. when $k_{v}$ is varied for a constant $k_{v} / k_{h}$. The same occurs for $a_{v}$ and $a_{h}$.

- When $k_{v} k_{h}$ is constant, the rate of change can be at least $0 \mathrm{~dB} / \mathrm{dec}$. by increasing $k_{v}$ when the $\alpha$ is less than the value $(=1.2)$ that is the intersection of both rates of change of $k_{v}$ and $k_{h}$, and by increasing $k_{h}$ when greater than that value. This also applies to a constant $a_{v} a_{h}$.

Next, we will determine the rate of change for the maximum output power $P_{\max }$ in the same manner. The normalized output power $P_{\max } / P_{a}$ shown in Fig. 8 is normalized by $P_{a}=\mu k_{v} a_{v} a_{h} \omega /(2 \pi) . P_{a}$ is also multiplied by 10 when $\mu, k_{v}, a_{v}$, or $a_{h}$ is multiplied by 10 . Thus, the rate of change of $P_{\max }$ becomes 10 times the rate of change of $P_{\max } / P_{a}$ as a function of $\alpha$ for $\mu, k_{v}$, and $a_{v}$; the reciprocal for $k_{h}$; and 10 times the reciprocal for $a_{h}$. Figure 12 shows the rates of change of $P_{\max }$ that were determined. From this

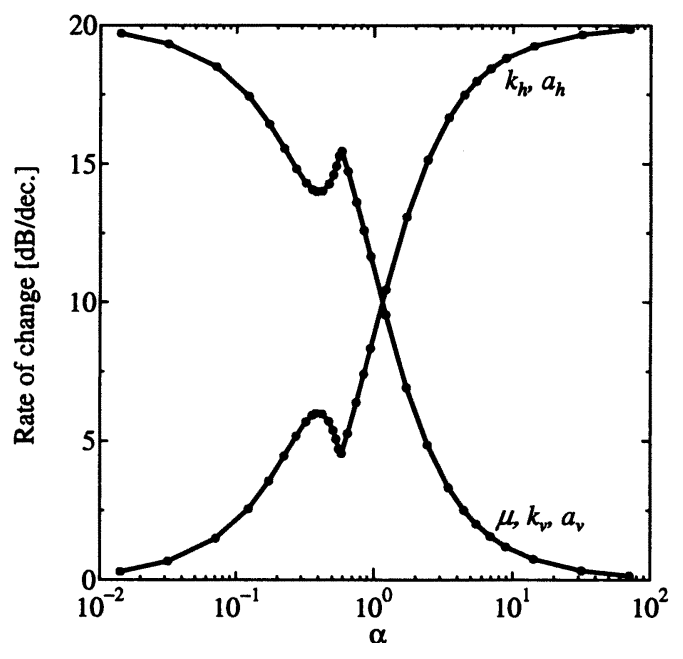

Fig. 11. Rate of change of $F_{\max }$. figure, we obtain the following knowledge about the maximum output power.

- Since the rates of change for all $\alpha$ 's are at least 0 $\mathrm{dB} / \mathrm{dec}$., the maximum output power always increases with increases in $\mu, k_{v}, k_{h}, a_{v}$, and $a_{h}$.

- Since the sum of the rates of change corresponding to $k_{v}$ and $k_{h}$ for all $\alpha$ is always $10 \mathrm{~dB} / \mathrm{dec}$., the rate of change of the maximum output power is always $10 \mathrm{~dB} / \mathrm{dec}$. when $k_{v}$ is varied for a constant $k_{v} / k_{h}$. On the other hand, this rate of change is always 20 $\mathrm{dB} /$ dec. for $a_{v}$ and $a_{h}$.

- When $k_{v} k_{h}$ is constant, the rate of change can be at least $0 \mathrm{~dB} / \mathrm{dec}$. when $k_{v}$ is increased when $\alpha$ is less than the value of the intersection $(=0.85)$ of both rates of change of $k_{v}$ and $k_{h}$, and $k_{h}$ is increased when greater than that value. On the other hand, when $a_{v} a_{h}$ is constant, since the rate of change with respect to $a_{h}$ always exceeds the rate of change with respect to $a_{v}$, the rate of change is always at least $0 \mathrm{~dB} / \mathrm{dec}$. by increasing $a_{h}$.

The above clearly showed the effects of changing each parameter. When we look at actual sliders, the ball slider is considered where $\alpha=0.2$ and $k_{v} / k_{h}=1$. In the silicon slider, $\alpha$ is roughly 1 to 5 , and $k_{v} / k_{h}$ is in the range of 5 to 15 . If we consider the knowledge gained, $k_{v}$ can be increased even if $k_{h}$ decreases for the ball slider, and a greater $k_{h}$ is preferred even if $k_{v}$ decreases for the silicon slider. A large $k_{v} / k_{h}$ for the silicon slider is considered because $k_{h}$ is decreased by the slip at the projection tips [2]. To increase the driving force and the output power, an intermediate shape between the cylindrical projection and the spherical projection is preferred. However, when a

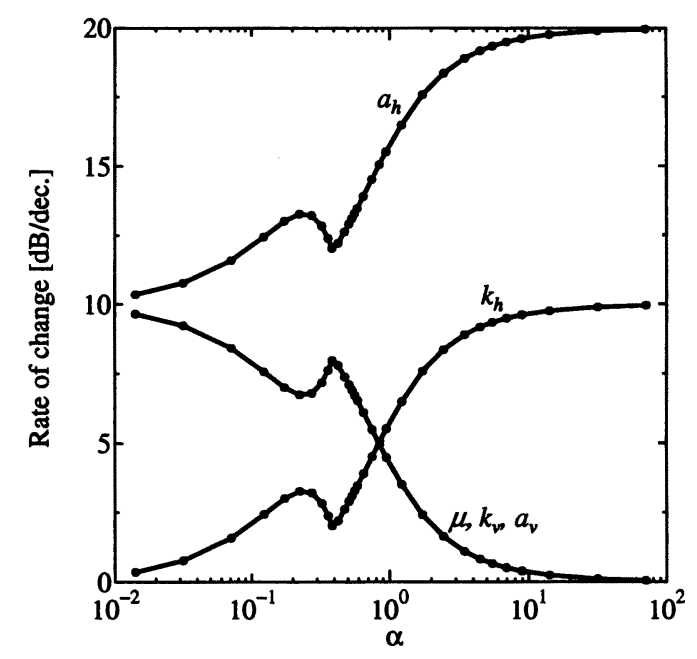

Fig. 12. Rate of change of $P_{\max }$. 
slider is actually designed, the sum of the driving forces generated by each projection becomes the driving force of the entire slider, we must consider not only the driving force per projection, but also the number of projections on the entire slider and the stable contact of projections.

Here, we will simply consider the effect when the static friction coefficient $\mu_{s}$ and the dynamic friction coefficient $\mu_{d}$ differ. From Figs. 11 and 12, the effect of changing $\mu$ on $F_{\max }$ and $P_{\max }$ increase for a small $\alpha$, and decrease for a large $\alpha$. When $\alpha$ is small, slip dominates the contact state, and stick dominates when $\alpha$ is large. In other words, in the state where $\alpha>10$, since the elastic force increases until there is little slip, the results hardly change at all even if the static friction coefficient is considered. Conversely, in the state where $\alpha<0.1$, since most of the contact states are slip, the results hardly change at all even if the static friction coefficient is considered. Thus, the effect due to considering the static friction coefficient for an intermediate $\alpha$ is most apparent, and $F_{\max }$ is calculated for $\mu_{d}=\mu$ and $\alpha=1$. The rate of increase of $F_{\text {max }}$ is limited to $1.1 \%\left(\mu_{s} / \mu_{d}=1.2\right)$ and $3.7 \%\left(\mu_{s} / \mu_{d}=1.4\right)$, and its effect is clearly small.

\subsection{The relationship between driving force and slip loss}

The efficiency of the surface acoustic wave motor can be considered to be the product of the conversion efficiency from the electrical energy to the vibration energy of the Rayleigh wave and the conversion efficiency from the vibration energy of the Rayleigh wave to the slider output. Most of the energy not converted from the vibration energy of the Rayleigh wave to the slider output is propagated as vibration energy, and a portion is lost in the friction drive surface. This indicates that the energy propagated as the vibration energy of the Rayleigh wave can be used to improve the conversion efficiency from the electrical energy to the vibration energy of the Rayleigh wave by using the energy circulation method [14]. The remaining loss in the friction drive surface is equivalent to the slip loss determined from the friction drive model. Since the generation of slip loss causes friction and affects durability, the relationship between the slip loss and the drive conditions must also be explained.

Figures 13 to 15 show the relationship between the driving force $F$ and the slip loss $L$. The slip loss is normalized by $P_{a}$ similar to the output power. We see from these figures that slip losses tend to vary greatly with $\alpha$. The slip loss tends to increase as $\phi$ decreases for $\alpha=0.1$, and tends to decrease as $\phi$ increases for $\alpha=10$. The reason is the slip loss increases without increasing the sticking region as the preload increases because the stator substrate can apply a too large driving force to the projections at the limit of the frictional force at $\alpha=0.1$. Conversely, for $\alpha=10$, the

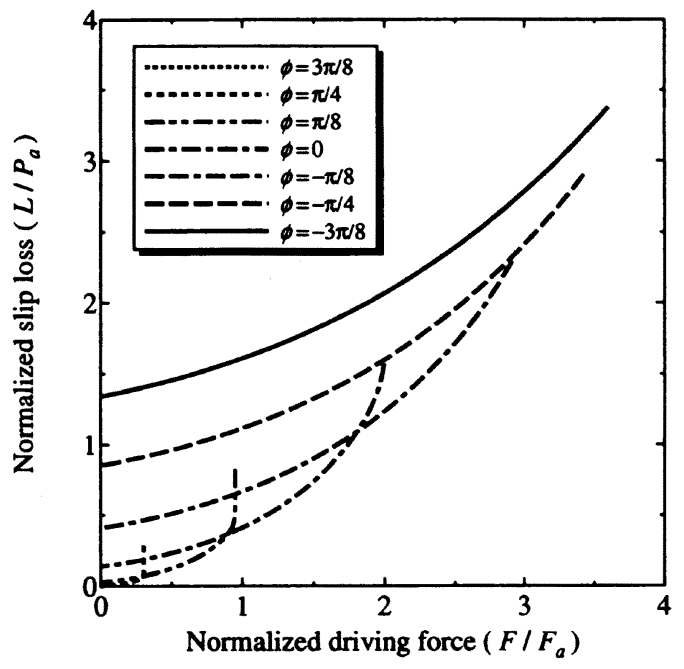

Fig. 13. Driving force-slip loss curves $(\alpha=0.1)$.

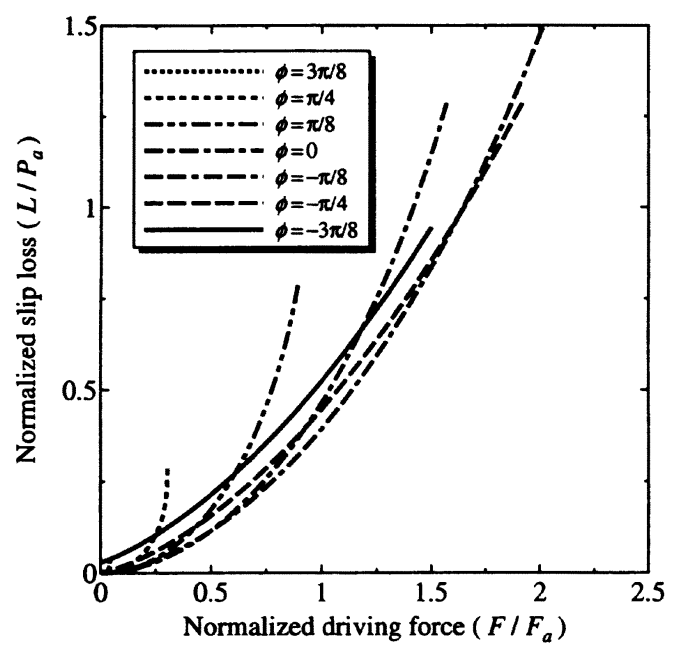

Fig. 14. Driving force-slip loss curves $(\alpha=1)$.

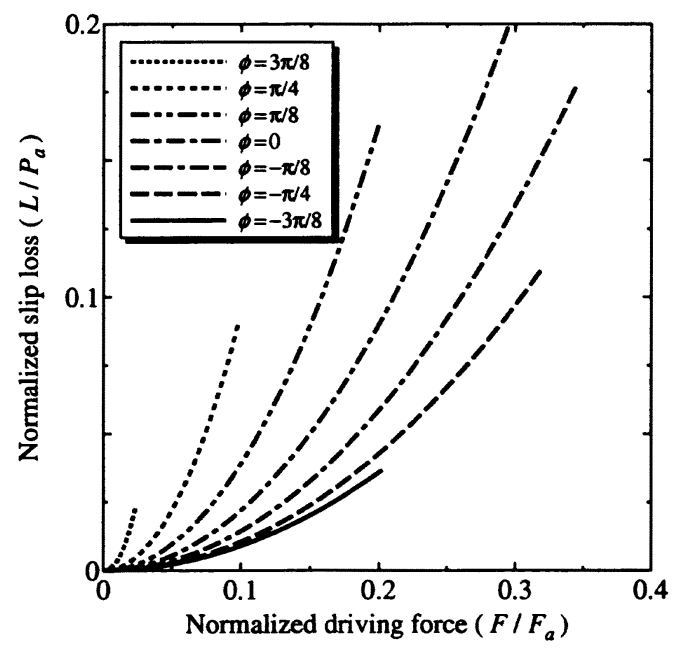

Fig. 15. Driving force-slip loss curves $(\alpha=10)$. 
sticking region is increased and the slip loss is decreased by applying the preload. For $\alpha=1$, the decrease in the slip loss due to the increase in the sticking region and the increase in the slip loss due to the increase in the preload are nearly the same, and the changes in the loss due to $\phi$ decrease.

\subsection{The relationship between driving force and efficiency}

The efficiency in the friction drive surface is defined as $\eta=P /(P+L)$. Figure 16 shows the efficiency when the output power is $P_{\max }$. It is seen that the efficiency is about 0.35 to 0.5 overall when driven under the condition of maximum output power, and maximizing the output power is not the desired state from the perspective of efficiency in the friction drive surface.

To determine the drive condition when efficiency is emphasized, Figs. 17 to 19 show the relationship between the driving force $F$ and the efficiency $\eta$. At $\alpha=10$, the efficiency improves as the driving force approaches 0 regardless of $\phi$. As $\alpha$ decreases, losses are generated successively even for driving force 0 from a small $\phi$. The efficiency reaches its maximum at the driving force between the 0 speed driving force and 0 . However, these results do not simply indicate that a large $\alpha$ or a small $\phi$ is superior from the perspective of efficiency. To further study the efficiency, we determined the maximum driving force for the efficiencies of $\eta=0.7,0.8$, and 0.9 while varying $\alpha$. Figure 20 shows these results. Figures 21 and 22 show $\phi$ and the slider speed, respectively, under the conditions obtaining the maximum driving force. These figures also show the results for the output power of $P_{\max }$ for reference. Figure 20 shows

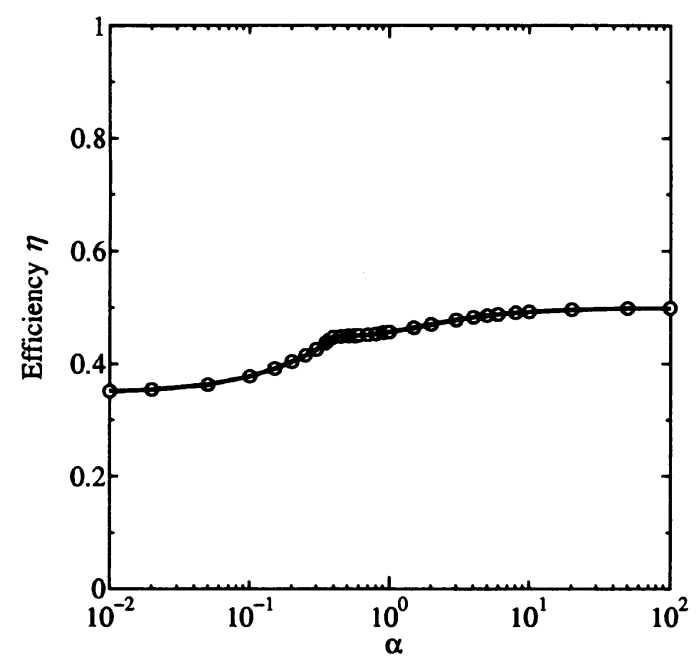

Fig. 16. Efficiency at output power $P_{\max }$.

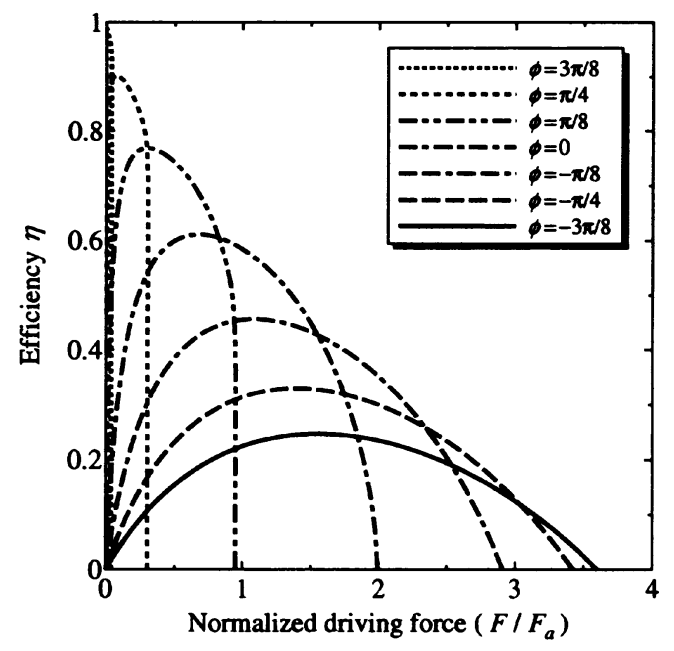

Fig. 17. Driving force-efficiency curves $(\alpha=0.1)$.

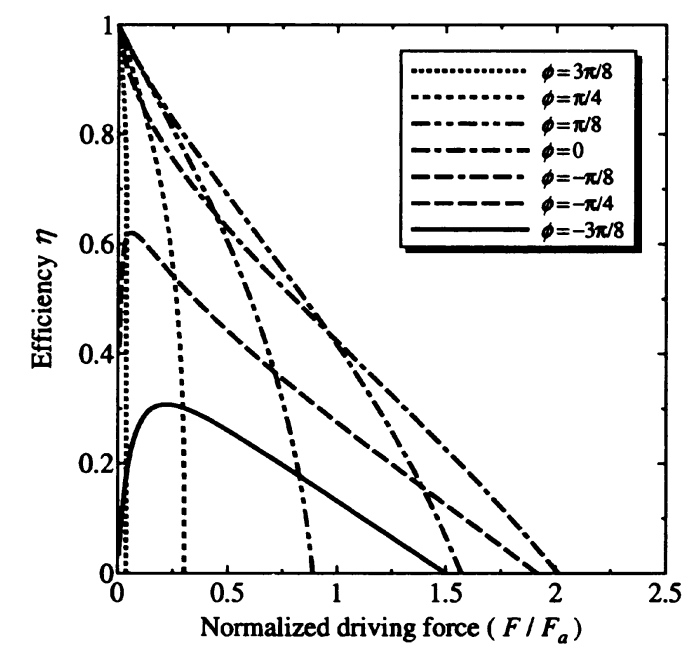

Fig. 18. Driving force-efficiency curves $(\alpha=1)$.

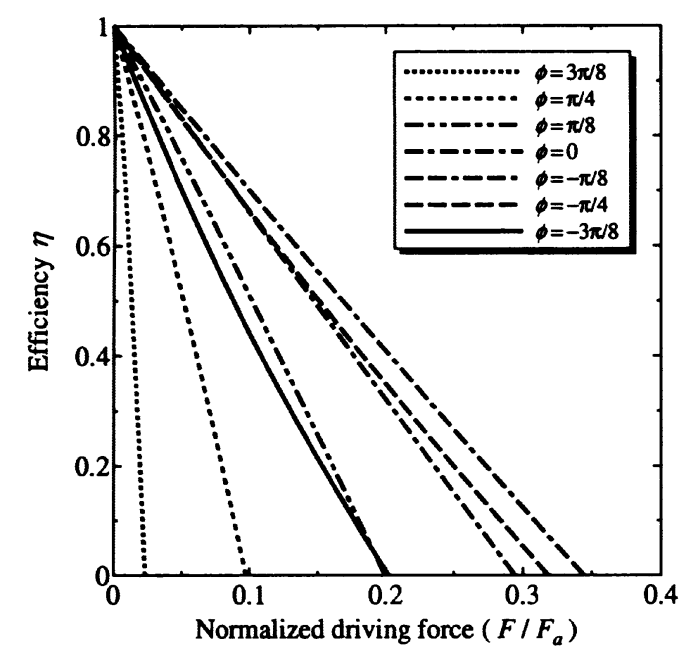

Fig. 19. Driving force-efficiency curves $(\alpha=10)$. 
the tendency for the normalized maximum driving force to decrease as the efficiency improves. The normalized maximum driving force basically increases as $\alpha$ decreases. However, there is little improvement in the driving force when $\alpha<1$ as the efficiency improves. When $\eta=0.9$, there is a peak near $\alpha=0.9$.

We see from Fig. 21 that with $\alpha=1$ as the boundary, $\phi$ increases as the efficiency improves below this boundary, but when large, $\phi$ becomes smaller than that in the condition of the output power $P_{\max }$ regardless of $\eta$. This indicates that when $\alpha$ is 1 or less, the preload must be small to improve the efficiency. Conversely, when $\alpha$ is greater than 1 , the preload must be increased based on the condition to achieve the output power $P_{\text {max }}$ to reduce the slip. Near $\alpha=1$, the $\phi$ where the output power is $P_{\max }$ nearly agrees with the $\phi$ where efficiency was emphasized. Therefore, the condition for the maximum output power and the condition emphasizing the slider speed can be switched by changing the slider speed.

We see from Fig. 22 that the slider speed increases for a high efficiency. However, at $\eta=0.9$, the slider speed obtaining the maximum driving force becomes about $90 \%$ of the vibration speed for a small $\alpha$ and about $40 \%$ of the vibration speed for a large $\alpha$. The reason is the no-load speed of the slider decreases because $\phi$ decreases for a large $\alpha$.

If the actual slider state is considered, the preload can be decreased for efficient driving by the ball slider $(\alpha=0.2)$. On the other hand, for the silicon slider $(\alpha=1$ to 5$)$, a preload can be added so that $\phi=0$ to $-\pi / 8$ results. If we also consider $\phi$ for the maximum driving force in Fig. 9, this preload will be positioned between the optimum preload with respect to the 0 speed driving force and the optimum

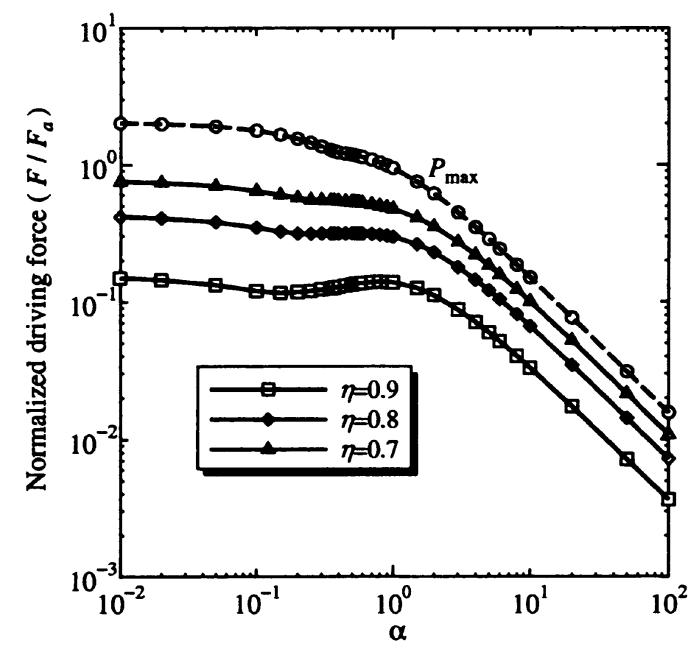

Fig. 20. Maximum driving force as a function of efficiency $\eta$.

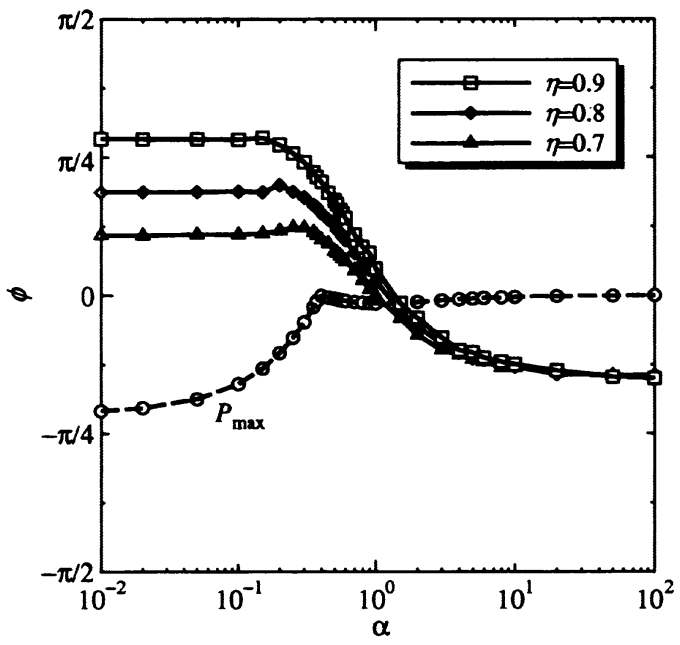

Fig. 21. $\phi$ for the maximum driving force as a function of efficiency $\eta$.

preload with respect to the output power. When the silicon slider is compared to the ball slider, we can consider the silicon slider to easily satisfy both the drive condition emphasizing the driving force and the output power and the efficient drive condition. However, this efficient drive only indicates a high percentage of output power in the energy lost in the contact surface. We must study not only whether $\eta$ increase when the decrease in wear is considered, but the materials of the stator substrate and the slider, and the projection shape.

We explained the effect of changing the drive conditions on the drive performance. We considered improving the performance of the surface acoustic wave motor by

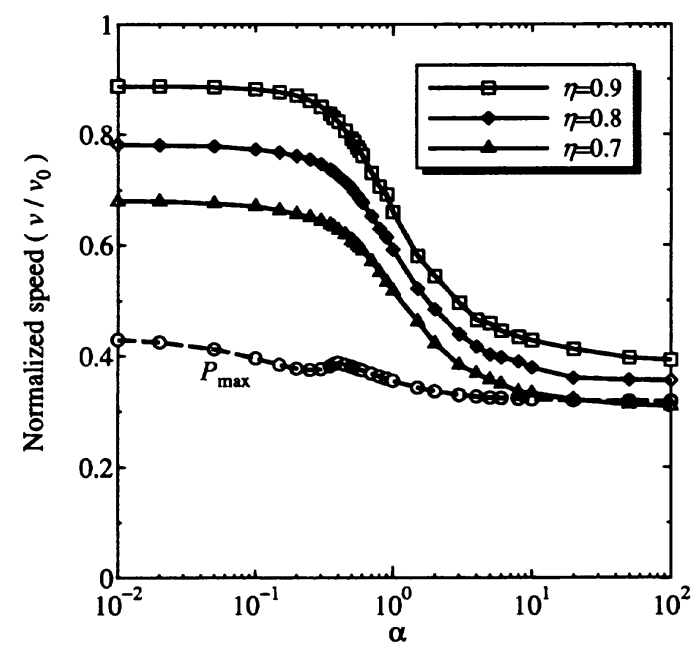

Fig. 22. Slider speed for the maximum driving force as a function of efficiency $\eta$. 
using these results to select design and drive conditions corresponding to the objective. However, attention must be paid to the application range of the model. When modeling the silicon slider described in Ref. 2, the effect of the slider ends is not considered because the projections are placed to infinity at equal spacing. Moreover, the fluctuations of the contact state of the projections and the deformations of the projections are not modeled. Therefore, we must examine the effects of exceeding the application range for modeling a design when the design is changed so that the slider size, stator substrate thickness, and projection size differ greatly from the current structure.

\section{Conclusion}

In this paper, we used the friction drive model which can distinguish the stick state from the slip state and analyzed the effect of changing each parameter on the drive performance. The results showed that the drive performance changed greatly by varying the rigidity of the contact surface and clearly showed the importance of the slider design. We then explained the drive conditions for maximizing the 0 speed driving force and output power and the achievable maximum values. Furthermore, we evaluated the efficiency of the friction drive surface and presented the drive conditions when emphasizing the efficiency, and the drive performance in that state. Based on the calculations of the effect of changing each parameter on the drive performance, we obtained predictions able to improve the 0 speed driving force and output power by using a projection shape which is an intermediate shape between the spherical projection and the cylindrical projection. Based on the knowledge we obtained, in the future, we plan to design a surface acoustic wave motor and optimize the drive performance.

Acknowledgment. This research was supported by a Grant-in-Aid for the Development of Innovative Technology of the Ministry of Education, Culture, Sports, Science and Technology.

\section{REFERENCES}

1. Asai K, Kurosawa M. Simulation model of surface acoustic wave motor considering tangential rigidity. Trans IEICE 2002;J85-A:1428-1439. (in Japanese)
2. Asai K, Kurosawa M. Simulation model of surface acoustic wave motor considering contact by projections arranged periodically. Trans IEICE 2003;J86A:1442-1452. (in Japanese)

3. Kurosawa M, Ueha S. Efficiency of ultrasonic motor using traveling wave. J Acoust Soc Japan 1988;44:40-46. (in Japanese)

4. Maeno T, Tsukimoto T, Miyake A. Finite-element analysis of the rotor/stator contact in a ring-type ultrasonic motor. IEEE Trans Ultrason Ferroelectr Freq Control 1992;39:668-674.

5. Hirata H, Ueha S. Characteristics estimation of a traveling wave type ultrasonic motor. IEEE Trans Ultrason Ferroelectr Freq Control 1993;40:402-406.

6. Zharii OY. An exact mathematical model of a travelling wave ultrasonic motor. 1994 IEEE Ultrasonics Symposium Proc, p 545-548, Cannes, France.

7. Hagood NW IV, McFarland AJ. Modeling of a piezoelectric rotary ultrasonic motor. IEEE Trans Ultrason Ferroelectr Freq Control 1995;42:210-224.

8. Aoyagi M, Tomikawa Y. Simplified equivalent circuit of ultrasonic motor and its application to estimation of motor characteristics. Jpn J Appl Phys 1995;34: 2752-2755.

9. Maeno T. Contact analysis of traveling wave type ultrasonic motor considering stick/slip condition. J Acoust Soc Japan 1998;54:305-311. (in Japanese)

10. Hagedorn P, Sattel T, Speziari D, Schmidt J, Diana G. The importance of rotor flexibility in ultrasonic traveling wave motors. Smart Mater and Struct 1998;7:352-368.

11. Le Moal P, Cusin P. Optimization of travelling wave ultrasonic motors using a three-dimensional analysis of the contact mechanism at the stator-rotor interface. Eur J Mech A/Solids 1999;18:1061-1084.

12. Ming Y, Peiwen Q. Performances estimation of a rotary traveling wave ultrasonic motor based on twodimension analytical model. Ultrasonics 2001;39: 115-120.

13. Sun D, Liu J, Ai X. Modeling and performance evaluation of traveling-wave piezoelectric ultrasonic motors with analytical method. Sensors Actuators A 2002;100:84-93.

14. Asai K, Kurosawa M, Higuchi T. Energy circulation methods for surface acoustic wave motor. Trans IEICE 2003;J86-A:345-353. (in Japanese) 
AUTHORS (from left to right)
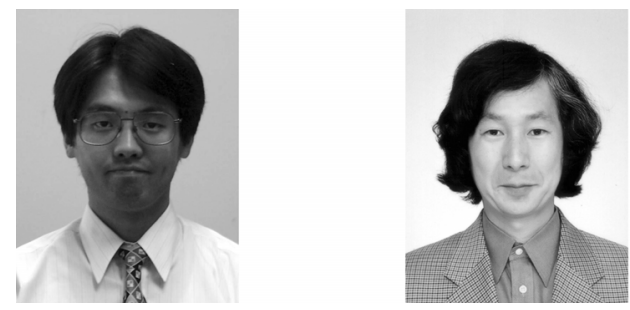

Katsuhiko Asai (member) received his B.S. and M.S. degrees in mechanical engineering from Tokyo Institute of Technology, Tokyo, in 1992 and 1994, respectively. He joined Matsushita Electric Industrial Co., Ltd., Osaka, Japan in 1994. He has been engaged in research and development of micro actuators. He is a member of the Japan Society of Mechanical Engineers.

Minoru Kuribayashi Kurosawa (member) received his B.Eng. degrees from Tokyo Institute of Technology, Tokyo, in 1982, 1984, and 1990, respectively. Beginning in 1984, he was a research associate of the Precision and Intelligence Laboratory, Tokyo Institute of Technology, Yokohama, Japan. From 1992, he was an associate professor at the Department of Precision Machinery Engineering, Graduate School of Engineering, The University of Tokyo. Since 1999, he has been an associate professor at the Department of Advanced Applied Electronics, Interdisciplinary Graduate School of Engineering, Tokyo Institute of Technology, Yokohama, Japan. His current research interests include ultrasonic motor, micro actuator, PZT thin film, SAW actuator, and single bit digital signal processing and its application to control system. Dr. Kurosawa is a member of the Acoustical Society of Japan, IEEE, the Institute of Electrical Engineers of Japan, and the Japan Society for Precision Engineering. 